\title{
Reverse Transcription Lesion-Induced DNA Amplification: An Instrument-Free Isothermal Method to Detect RNA
}

\author{
B. Safeenaz Alladin-Mustan ${ }^{a}$, Yuning Liu ${ }^{a}$, Yimeng Li ${ }^{a}$, Daria R. Q. de Almeida ${ }^{a}$, Jesse Yuzik ${ }^{a}$, Camilla F. \\ Mendes ${ }^{a}$ and Julianne M. Gibbs $* a$ \\ ${ }^{a}$ Department of Chemistry, University of Alberta, Edmonton, Alberta, Canada, T6E 2G2.
}

\begin{abstract}
One challenge in point-of-care diagnostics is the lack of room-temperature methods for RNA detection based on enzymatic amplification and visualization steps. Here we perform a reverse transcription ligase chain reaction using our isothermal lesion induced DNA amplification (LIDA) technique that can be tuned to operate at any desired temperature. Using RNA-triggered LIDA, we can detect as little as 100 attomoles target RNA and can distinguish RNA target from total cellular RNA. Finally, we demonstrate that the resulting DNA amplicons can be detected colorimetrically, also at room temperature, by rapid, targettriggered disassembly of DNA-modified gold nanoparticles. This integrated amplification/detection platform requires no heating or visualization instrumentation, which is an important step towards realizing instrument-free POC testing.
\end{abstract}

\section{Graphical abstract}

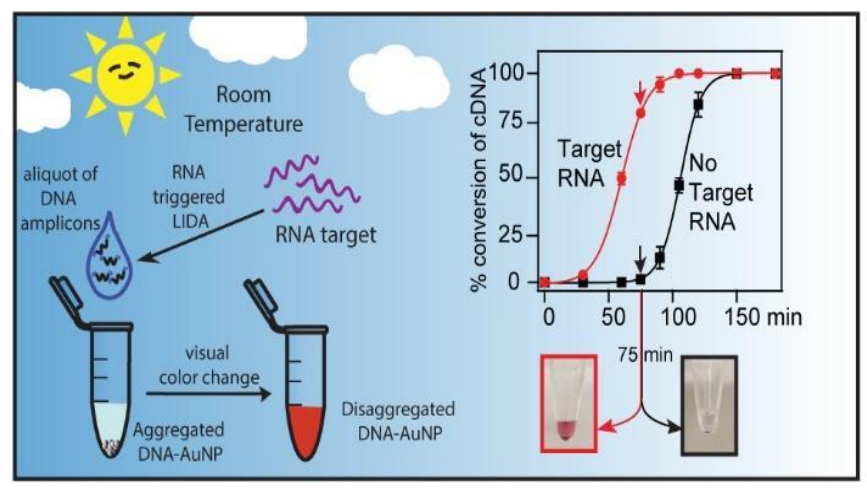




\section{Keywords}

Lesion-induced DNA amplification, DNA modified gold nanoparticles, RNA detection, colorimetric method, room temperature amplification and detection

\section{Abbreviations}

LIDA, DNA-AuNP, POC

\section{Introduction}

The use of ribonucleic acid (RNA) sequences as a biomarker for the diagnosis of a range of diseases has gained attention over the last few decades.[1, 2] For example, many infectious viruses such as HIV[3], Ebola[4], hepatitis C[3], Zika[4, 5], Covid-19[6, 7] have RNA as their genetic material rather than DNA. Additionally, messenger RNA (mRNA) are present in bacterial cells in larger abundance than DNA,[1] so mRNA can be a useful biomarker for bacterial detection. $[8,9]$ Moreover, as protein expression levels are mirrored in mRNA concentration, mRNA biomarkers are also useful for detecting cell abnormalities.[10, 11] Finally, microRNA (miRNA) is a class of naturally occurring RNA of short length,[12] and the abundance of specific sequences can be correlated with various diseases such as cancer, diabetes, heart failure and diabetes mellitus amongst others.[2, 13-15] Thus, the development of a simple, rapid and sensitive RNA detection platform that can be performed with minimal training and in limited resource settings is key for

early disease diagnosis, prognosis, and treatment especially in low income countries where infectious diseases are more prevalent.[16] However, one major problem of nucleic acid based diagnostics is the low concentration of nucleic acids biomarker in the biological sample, be it blood, urine or saliva. $[12,13,17]$ Therefore, a key step prior to detection of the biomarker is to amplify the nucleic acid.[17] Yet, methods for the enzymatic amplification of nucleic acids without the use of a heating element are rare $[18,19]$, although equipment- or instrument-free platforms are ideal for point-of-care (POC) diagnostics as outlined by the World Health Organisation.[20, 21] 
Many techniques for RNA biomarker-triggered nucleic acid amplification have been developed but reverse transcription polymerase chain reaction (RT-PCR) remains the gold standard.[3-5] It is a two-step process that relies on transcribing the RNA into a complementary DNA (cDNA) using the reverse transcriptase enzyme followed by that cDNA undergoing exponential enzymatic amplification by a PCR reaction. RT-PCR has shown great sensitivity, but it requires multiple steps, two enzymes, highly trained personnel and a thermocycler, which makes it time consuming and expensive. [5, 14, 22, 23] Once more this method is not well suited to the detection of short sequences such as that of miRNA, which are typically $19-23$ bases long, so complex primers have to be designed to accommodate the short target.[14, $22,24,25]$ As such efforts have been made to develop ways to lengthen the RNA or CDNA prior to the PCR step adding another layer of complexity and cost to the assay.[12, 25-27] To simplify the instrumentation requirements, much research has focused on developing isothermal amplification techniques like RT-loop mediated isothermal amplification (LAMP) $[25,28]$ and rolling circle amplification (RCA)[29] for POC diagnostics.

One challenge of isothermal methods like RT-LAMP and RCA commonly used in POC applications, is that they often require more than one enzyme and higher temperatures, the latter necessitating the use of heating instrumentation.[28-30] For example, RT-LAMP, like RT-PCR, utilizes reverse transcriptase in the first step to transcribe RNA into cDNA,[28] while for RCA an RNA-templated DNA ligation is performed with ligase to generate a circular template. $[29,30]$ Next the cDNA is liberated from the RNA by digestion or nicking enzymes followed by amplification by polymerase.[11, 28] These sequential steps to form the cDNA and then amplify it often lead to the use of multiple temperatures even when isothermal amplification methods are employed[31]. To overcome these problems and present a complementary approach to RT-polymerase based methods, we describe a simple, instrument-free method for detecting RNA that is completely performed at room temperature, uses only one enzyme (a ligase) and colorimetric detection. Although examples exist for sensitive RNA detection that require no heating element,[32] the 
detection step often requires significant instrumentation, unlike our method that requires only eppendorf tubes and reagents to proceed.

Previously we reported the development of lesion-induced DNA amplification (LIDA), which was capable of rapid, exponential amplification of different 18 nucleotide DNA target sequences by incorporating a destabilizing abasic lesion into one of the target-complementary primers, or probes, in a ligase chain reaction.[33-35] With this approach, as little as $140 \mathrm{fM}$ (2.1 attomoles) of target DNA was detected following a two-step serial amplification procedure.[35] In more recent work, it was revealed that adding a second destabilizing lesion consisting of a mismatch or another abasic group allowed the replication temperature to be tuned from $18-30{ }^{\circ} \mathrm{C}$ for a particular target sequence. Using the optimal probe sequences, target DNA was amplified on the benchtop without any heating source or equipment.[33] Regarding the visualization step, our group has also developed a rapid, room-temperature method for detecting single-stranded DNA using DNA-modified gold nanoparticle aggregate disassembly.[36] Herein we combine these two approaches and add an additional step to facilitate both amplification and detection triggered by a RNA target sequence. This RNA-triggered LIDA uses only one enzyme and proceeds entirely at $28^{\circ} \mathrm{C}$. Using this modified LIDA followed by CDNA-initiated nanoparticle disassembly, we can distinguish 105 amol target RNA in the presence of cellular RNA within three hours by colorimetric detection.

\section{Experimental}

\subsection{Reagents and materials}

Urea (cat. \# 9912), tetramethylethylenediamine (TEMED), ammonium persulphate (cat. \# AMP 001), tris base (cat. \# BP1521), sodium dodecyl sulfate (SDS), and sodium azide were purchased from Fisher. Gold (III) chloride trihydrate, sodium chloride, sodium citrate tribasic dihydrate and dithiothreitol were bought from Sigma Aldrich. For polyacrylamide gel synthesis, the 40\% acrylamide/bis solution 19:1 (cat. \# 161-0144) was purchased from Bio-Rad. Nuclease free water (NFW) was purchased from Integrated DNA 
Technologies was used for all experiments. All RNA oligonucleotides were bought from Integrated DNA Technologies (IDT, lowa, USA) and all DNA oligonucleotides were synthesized using phosphoramidites and reagents from Glen Research (Sterling, VA). The enzyme, T4 DNA ligase was bought from New England Biolabs while the human lungs total RNA (\#AM7968) and E. coli total RNA (\#AM7940) was bought from Fisher Scientific.

\subsection{Synthesis and characterization of DNA}

All the DNA was synthesized following manufacturer's instruction using an Applied Biosystems Model 392 DNA/RNA Synthesizer. Some of the DNA strands had special modifications such as fluorescein, 5'phosphate, $5^{\prime}$ or $3^{\prime}$ thiol and the abasic lesion. Fluorescein-dT phosphoramidite and 3' Thiol Modifier C3 S-S CPG (Glen Research, Sterling, VA) were used to incorporate the fluorescein and the $3^{\prime}$ thiol, respectively, while Chemical Phosphorylating Reagent II and Thiol Modifier C6 (Glen Research, Sterling, VA) were used to add the $5^{\prime}$-phosphate group and the thiol group on the $5^{\prime}$ end of the DNA oligonucleotide, respectively. Furthermore, the abasic group was incorporated using a dSpacer CE phosphoramidite (Glen Research, Sterling, VA).

All synthesized DNA was purified with the DMT-On protocol using Glen-Pak cartridges according to the manufacturer's instruction. The strands were characterized by MALDI-TOF under a linear negative mode using a Voyager Elite time of flight-mass spectrometer (Applied Biosystems, Foster City, CA) following our previous work.[35] Purity was assessed by visualizing the oligonucleotides with StainsAll reagent after it has been run on a $15 \%$ polyacrylamide denaturing gel using the ImageQuant RT ECL Imager from GE Healthcare Life Science (with the fluorescein filter). DNA and RNA strand concentrations were determined from the absorbance at $260 \mathrm{~nm}$ using OligoCalc (http://biotools.nubic. northwestern.edu/OligoCalc.html) to determine the extinction coefficients of each strand.

\subsection{Preparation of DNA-modified AuNPs}


DNA functionalized gold nanoparticles were prepared according to our previous work with some modifications. [36] Specifically, for each batch of DNA-modified gold nanoparticles, the thiolated DNA probe strand ( $15 \mathrm{nmol}$ ) after deprotection by DTT following the manufacturer's protocol was immediately added to a citrate-capped gold nanoparticle suspension ( $1 \mathrm{~mL}$ of the synthesized gold nanoparticles). This mixture was then salted up to $0.2-0.3 \mathrm{M} \mathrm{NaCl}$ following our previous work[36] while maintaining a constant concentration of $10 \mathrm{mM}$ phosphate buffer (0.01 wt\% SDS, $0.05 \mathrm{wt} \% \mathrm{NaN}_{3}, \mathrm{pH} 7$ ).

Each DNA-modified AuNP (150 fmol) and linker DNA (6 pmol) were combined and topped up to $10 \mu \mathrm{L}$ with $0.2 \mathrm{M} \mathrm{NaCl}$ in the above mentioned $10 \mathrm{mM}$ phosphate buffer and allowed to aggregate overnight. Newly synthesized DNA and AuNP aggregates were aliquoted and lyophilized. For the low target concentration assay, a reduced DNA-AuNP was prepared: 50 fmol DNA-modified AuNP and 2 pmol linker DNA was used.

\subsection{RNA transcription LIDA protocol}

For all of the RNA target-triggered experiments except for the target-variation experiments, the following procedure was used. Single-stranded RNA (105 fmol), probe DNA-Ilb (41 pmol) and DNA-Ila (41 pmol), 2000 CEU T4 DNA ligase, 50 mM TRIS- $\mathrm{HCl}, 10$ mM MgCl 2 and $10 \mu \mathrm{M}$ ATP were combined in a 600$\mu \mathrm{L}$ microtube (total volume of $7.5 \mu \mathrm{L}$. The reaction was allowed to react at $28{ }^{\circ} \mathrm{C}$ for 15 minutes unless otherwise stated. The non-templated reaction, which is the control (C) was performed similarly without the target (NFW was used instead of RNA solution). Following 15 min wait time, $7.5 \mu \mathrm{L}$ of a master mix comprising of DNA-Ib (41pmol), dDNA (20.5 pmol), F-DNA-Ia (20.5 pmol), ATP (3 $\mu \mathrm{L}, 100 \mathrm{mM})$ and T4 DNA ligase buffer ( $3 \mu \mathrm{L}$, from New England Biolabs). The reaction tubes were placed again in the thermal incubator at $28{ }^{\circ} \mathrm{C}$ unless otherwise noted. Aliquots of the bulk reaction were then collected at several time points. Reaction was stopped in each aliquot with $2 \mu \mathrm{L}$ of a $0.5 \mathrm{M}$ EDTA/sucrose/ bromophenol blue mixture. After all the data points were collected, the samples were run through $15 \%$ denaturing PAGE.

For target variation experiments a lower concentration of probes was used as this has been shown to increase our ability to discriminate from the background-triggered process.[35] Specifically, various RNA 
concentrations in the first step were $14 \mathrm{nM}(105 \mathrm{fmol}), 1.4 \mathrm{nM}(10.5 \mathrm{fmol}), 140 \mathrm{pM}(1.05 \mathrm{fmol})$ and $14 \mathrm{pM}$ (105 amol) while the fluorescent probe F-DNA-la final concentration after both steps was $0.35 \mu \mathrm{M}$ (5.38 pmol per $15 \mu \mathrm{L})$ and all other probes had a final concentration of $0.7 \mu \mathrm{M}(10.3$ pmol per $15 \mu \mathrm{L})$ unless otherwise stated. Also, a longer wait time of 1 hour was employed for the RNA templated ligation.

\section{Results and Discussion}

\subsection{Design of isothermal RNA-triggered LIDA}

To achieve rapid isothermal RNA detection using LIDA, the RNA is first transcribed into a cDNA using a ligation strategy rather than a reverse transcriptase (as compared to RT-PCR or RT-LAMP). In the second step, the cDNA is amplified in a cross catalytic fashion using lesion-induced turnover in a ligase chain reaction as shown in Figure 1 . Specifically, in step A, the target RNA hybridizes with two complementary DNA primers, or probes, (DNA-Ila and DNA-IIb) to form an RNA-cDNA nicked duplex, which is then ligated by T4 DNA ligase, the same enzyme used for DNA amplification by LIDA (Figure 1). This RNA-cDNA product duplex must be denatured, which can be achieved using various strategies like applying heat,[37] using a nuclease enzyme (example DSN or RNase H)[38-40] or by toehold-mediated strand displacement[29]. Since the goal was to have a simple, cost effective and isothermal assay, the latter option was selected. Displacement DNA (dDNA) was designed to hybridize with the RNA target sequence, including an overhang region (i.e. toehold) that stemmed from the use of DNA primers in the first step that were shorter than the RNA target (Figure 1). As the resulting RNA-dDNA duplex was designed to be more stable than the RNA-cDNA duplex, displacement should be spontaneous. Once liberated, the cDNA sequence can hybridize to two complementary probes (Figure 1, cycle B): one that contains an abasic site (oval) and the other a mismatch (square) as well as a fluorescent label (star). After templated ligation of these probes, the newly formed destabilizing template DNA (referred to as F-DNA as it contains a fluorescent label) spontaneously dissociates from the cDNA due to the presence of the destabilizing groups. A second reaction (Figure 1, cycle C) then uses this destabilizing strand to template the ligation of two other probes 
(the same probes in step A). The result is a copy of the cDNA (Figure 1, cycle C). This cross-catalytic replication of cDNA and F-DNA continues until all the probes are consumed.

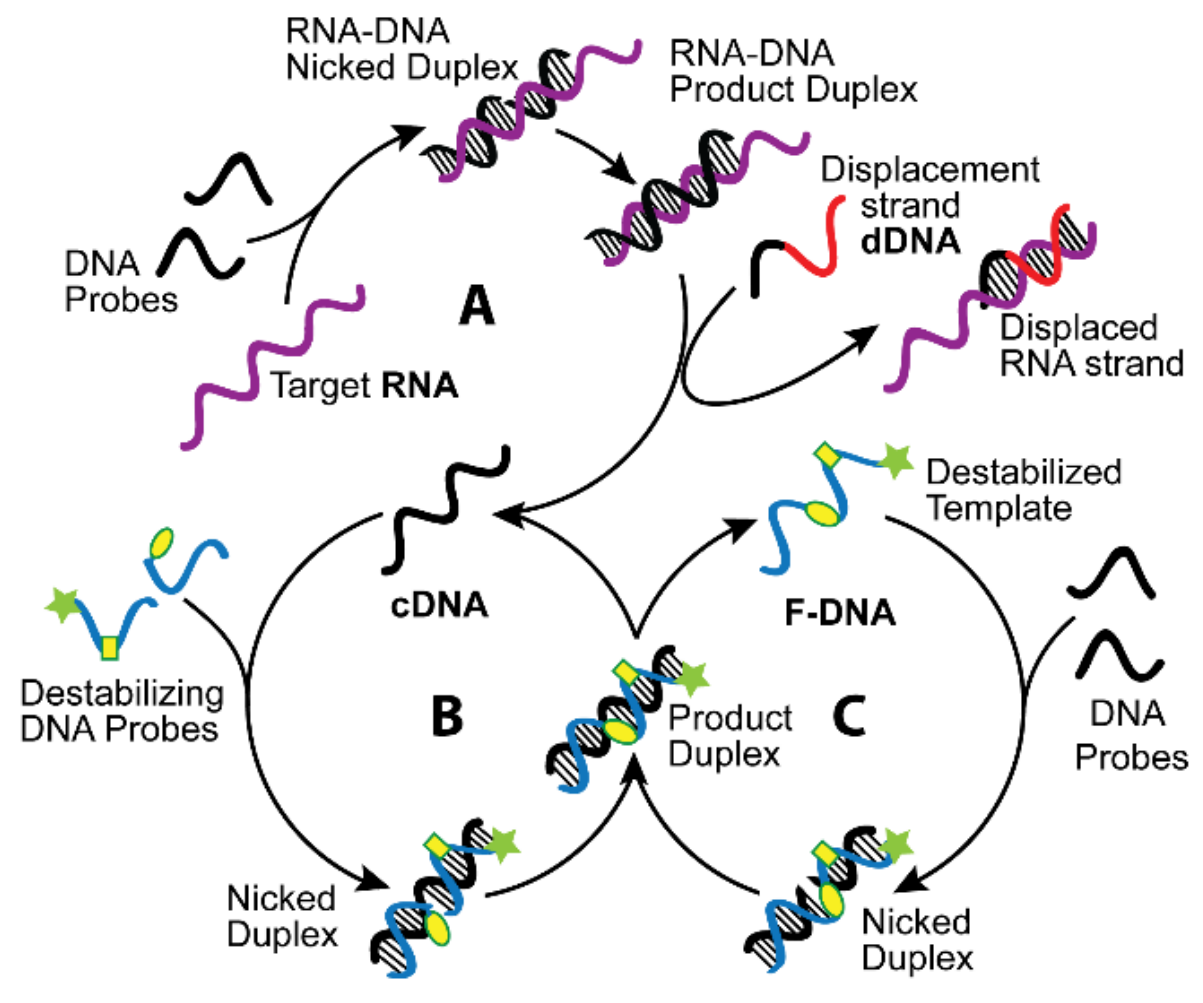

Fluorescein $\bigcirc$ Abasic Group $\square$ A : C mismatch

Figure 1. Schematic of reverse transcription lesion-induced DNA amplification (RT-LIDA). First the RNA target is transcribed into a cDNA strand, which is then amplified isothermally by LIDA after strand displacement by dDNA. Black probes: DNA-Ila and DNA-Ilb; Blue destabilizing probes: DNA-la and DNAIb; Oval: abasic lesion; Square: A:C mismatch; Star: fluorescein label.

3.2. Optimization of RNA transcription-lesion induced DNA amplification

\subsubsection{Influence of ATP concentration in the RNA-templated synthesis of cDNA}

Landegren and co-worker reported that RNA-templated ligation using T4 DNA ligase works best at low ATP concentration $(\sim 10 \mu \mathrm{M})$.[41] In contrast, our normal LIDA reaction is performed at an ATP concentration of $1 \mathrm{mM}$. Thus, to optimize the concentration of ATP, the RNA-templated ligation step 
(Figure 1, step A) was investigated separately for different ATP concentrations: $10 \mu \mathrm{M}, 100 \mu \mathrm{M}$ and $1 \mathrm{mM}$. For proof of concept, a 27-base long synthetic RNA sequence mimicking a miRNA (1.4 $\mu \mathrm{M})$ was incubated with $2.8 \mu \mathrm{M}$ of DNA-Ila and $1.4 \mu \mathrm{M}$ of fluorescently labelled DNA-Illb at varying ATP concentrations and $28{ }^{\circ} \mathrm{C}$. As expected, $>90 \%$ ligation was observed within 90 minutes using $10 \mu \mathrm{M}$ ATP based on polyacrylamide gel electrophoresis (PAGE) as compared to $100 \mu \mathrm{M}$ and $1 \mathrm{mM}$ ATP, which gave only $46 \%$ and $23 \%$ ligation, respectively (Figure S1A). The time course of the ligation reaction with $10 \mu \mathrm{M}$ ATP based on PAGE analysis is shown in Figure S1B. These results confirmed that a lower concentration of ATP was required for RNA-templated DNA ligation as compared with the DNA-templated reaction to avoid premature AMP reloading of the enzyme owing to the slower ligation rate in the presence of an RNA template.[41]

\subsubsection{Tuning the temperature of cDNA-initiated LIDA}

To have an isothermal assay, both the RNA-templated ligation and LIDA of cDNA should work at the same temperature, ideally room temperature. However, the cross catalysis of this cDNA sequence by LIDA worked best at $37^{\circ} \mathrm{C}$ when only one destabilizing group was incorporated into the probe sequence (Figure $\mathrm{S} 2 \mathrm{~A}$, supporting information). As mentioned, our previous work showed that the replication temperature in LIDA can be tuned by the introduction of a mismatch to the system, which is less destabilizing than an abasic group.[33] Thus, a T base was switched into a C base in one of the probes (DNA-la), which resulted in an A:C mismatch (square, Figure 1). This combination of destabilizing elements allowed us to lower the temperature from $37^{\circ} \mathrm{C}$ to $28^{\circ} \mathrm{C}$ (Figure S2B). 

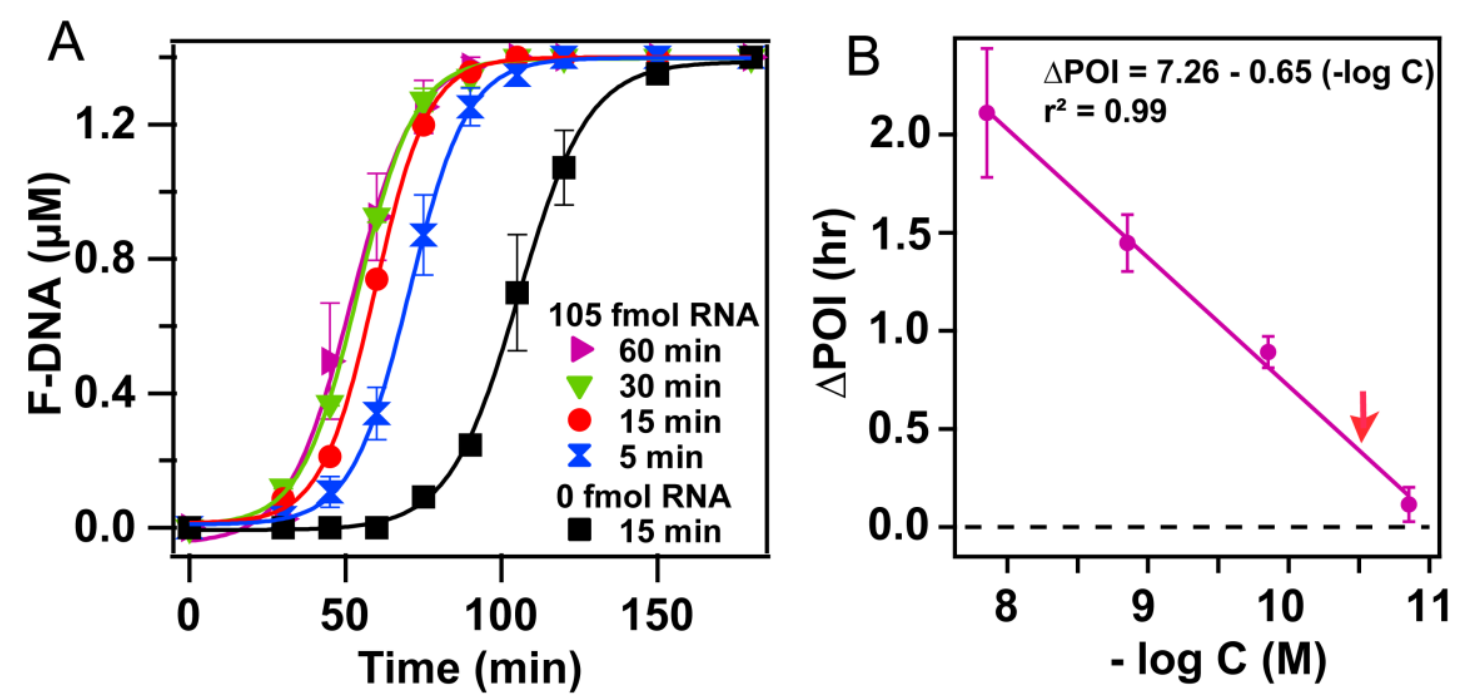

Figure 2. A) Kinetics of cross-catalytic formation of F-DNA at $28{ }^{\circ} \mathrm{C}$ initiated by cDNA formed from the templated ligation of $105 \mathrm{fmol}$ (purple, green, red, blue traces) or 0 fmol (black trace) RNA target. The legend refers to the reaction time of step one in RT-LIDA, the RNA-templated ligation step. Probe concentrations: $1.4 \mu \mathrm{M}$ F-DNA-la; $2.8 \mu \mathrm{M}$ DNA-Ib; $2.8 \mu \mathrm{M}$ DNA-Ila; $2.8 \mu \mathrm{M}$ DNA-IIb; $1.4 \mu \mathrm{M}$ dDNA. B) Difference in point of inflection between the kinetic traces of LIDA with no initial RNA target and various initial RNA target concentrations ( $\triangle \mathrm{POI}$ ). Probe concentrations: $0.35 \mu \mathrm{M}$ F-DNA-la; $0.7 \mu \mathrm{M}$ DNA-Ib; $0.7 \mu \mathrm{M}$ DNA-IIa; $0.7 \mu \mathrm{M}$ DNA-IIb; $1.4 \mu \mathrm{M}$.

\subsubsection{Combining RNA transcription and LIDA}

Next, the RNA-templated ligation step was merged with LIDA of cDNA using strand displacement to link both steps and maintain a constant temperature. First, RNA-templated DNA ligation was initiated by $105 \mathrm{fmol}(14 \mathrm{nM})$ target RNA in the presence of two probes (DNA-Ila and DNA-IIb), T4 DNA ligase, and 10 $\mu \mathrm{M}$ ATP. We also explored the background triggered process that leads to spontaneous LIDA in the absence of any target[35] by combining the two probes, enzyme and ATP and allowing them to incubate for 15 minutes (Figure 2A, 0 fmol RNA). After various wait times for the reaction mixtures containing target RNA as well as the negative control, more ATP was added to make up a concentration of $\sim 1 \mathrm{mM}$ ATP along with the two destabilizing probes (F-DNA-la and DNA-Ib) and the dDNA to liberate the cDNA from the 
RNA:cDNA duplex. The time trace of the resulting cross-catalytic formation of F-DNA (the complement of cDNA) by LIDA was followed by PAGE (Figure 2A). With 105 fmol RNA target and various wait times for the first step, LIDA proceeded faster than in the absence of RNA target (Figure 2A, $105 \mathrm{fmol}$ vs $0 \mathrm{fmol}$ ). This suggested that the cDNA had successfully been generated, displaced from the RNA:cDNA product duplex, and engaged in the cross catalytic reaction. Moreover, $60 \mathrm{~min}, 30 \mathrm{~min}$ and $15 \mathrm{~min}$ wait times for the first step of RNA-templated formation of CDNA did not exhibit significant differences in the LIDA kinetic profiles, so $15 \mathrm{~min}$ was taken as our optimized wait time for the first step of the reaction. As a control experiment, we performed the two-step process under the same conditions but the strand displacement DNA (dDNA) was not added at the beginning of the second step, and only backgroundtriggered LIDA was observed, indicating that no liberation of cDNA occurred in the absence of dDNA (Figure S3).

\subsection{Sensitivity of the assay}

With these optimized conditions, reverse transcription LIDA was performed with different initial concentrations of RNA (Figure S4), albeit at lower probe concentrations, which has been shown to slow down the background reaction compared with the templated process.[35] To determine the sensitivity of our assay, we determined the point-of-inflection (POI) from a sigmoidal fit to each kinetic trace. We then took the difference between the POI for the kinetic trace with RNA target and that from the reaction containing no RNA target (i.e., the background-triggered process) (Figure 2B). The $\triangle \mathrm{POI}$ versus the logarithm of initial RNA concentration $(-\log C)$ gave a linear trend with a line-of-best fit of $\triangle P O I=7.3 \pm 0.3$

$-0.65 \pm 0.03(-\log C),\left(r^{2}\right.$-value $\left.=0.99\right)$. From the line of best fit, we calculated a $-\log C$ value of $11.2 \pm 0.7$ for $\triangle \mathrm{POI}$ of $\mathrm{O}$ (the $\mathrm{x}$-intercept), which corresponds to the concentration when the templated reaction cannot be distinguished from the background-triggered process. The red arrow Figure 2B marks the upper limit of this value (- $\log C$ of 10.5$)$, which is equal to a concentration of $32 \mathrm{pM} \mathrm{(240} \mathrm{amol)} \mathrm{and} \mathrm{is} \mathrm{a} \mathrm{conservative}$ estimate of the limit of detection using PAGE and RT-LIDA. We note, however, that we could discriminate 
as low as $14 \mathrm{pM}$ (105 amol) initial RNA target and the background-triggered process based on a discernable $\triangle \mathrm{POI}$ (Figure 2B).

These results suggest reverse transcription lesion-induced DNA amplification has a similar sensitivity to our DNA-based assay indicating that transcribing the RNA into cDNA did not impact the overall cross catalysis reaction.[35] This similarity also implies that a serial ligation amplification procedure could be used to detect as low as 2 amol of initial RNA as shown in our previous work.[35] However, this procedure does add time to the assay. As our current sensitivity is suitable for microRNA target concentrations relevant to disease states (vide infra), [42] we did not optimize it any further.

\subsection{Selectivity of the assay}

To assess the selectivity of the assay, we compared RNA-triggered LIDA using three different RNA sequences including the complementary target RNA (matched), a one-base mismatched RNA and a random RNA sequence of the same length. Figure 3A exhibits the concentration of F-DNA formed at 60 $\min , 75 \mathrm{~min}$ and $90 \mathrm{~min}$ initiated by these different RNA sequences. As expected, there was a significant difference in F-DNA formed between the matched RNA strand (Figure 3A, red trace) compared to the mismatched (Figure 3A, blue trace) and random (Figure 3A, green trace) target. Moreover, the results from the mismatched and random strand were within error to that of the experiment that had no RNA target (Figure 3A, black trace) for all of the three time points. This suggested that only background (bluntend) ligation leading to the background-triggered LIDA as opposed to RNA-templated ligation in the presence of the non-target RNA strands. Additionally, a ligation reaction in which matched RNA, mismatched and random RNA were combined in one tube was conducted (Figure S5). An F-DNA amount similar to that from the matched RNA was observed, indicating the presence of random strands at these concentrations did not affect our assay. 

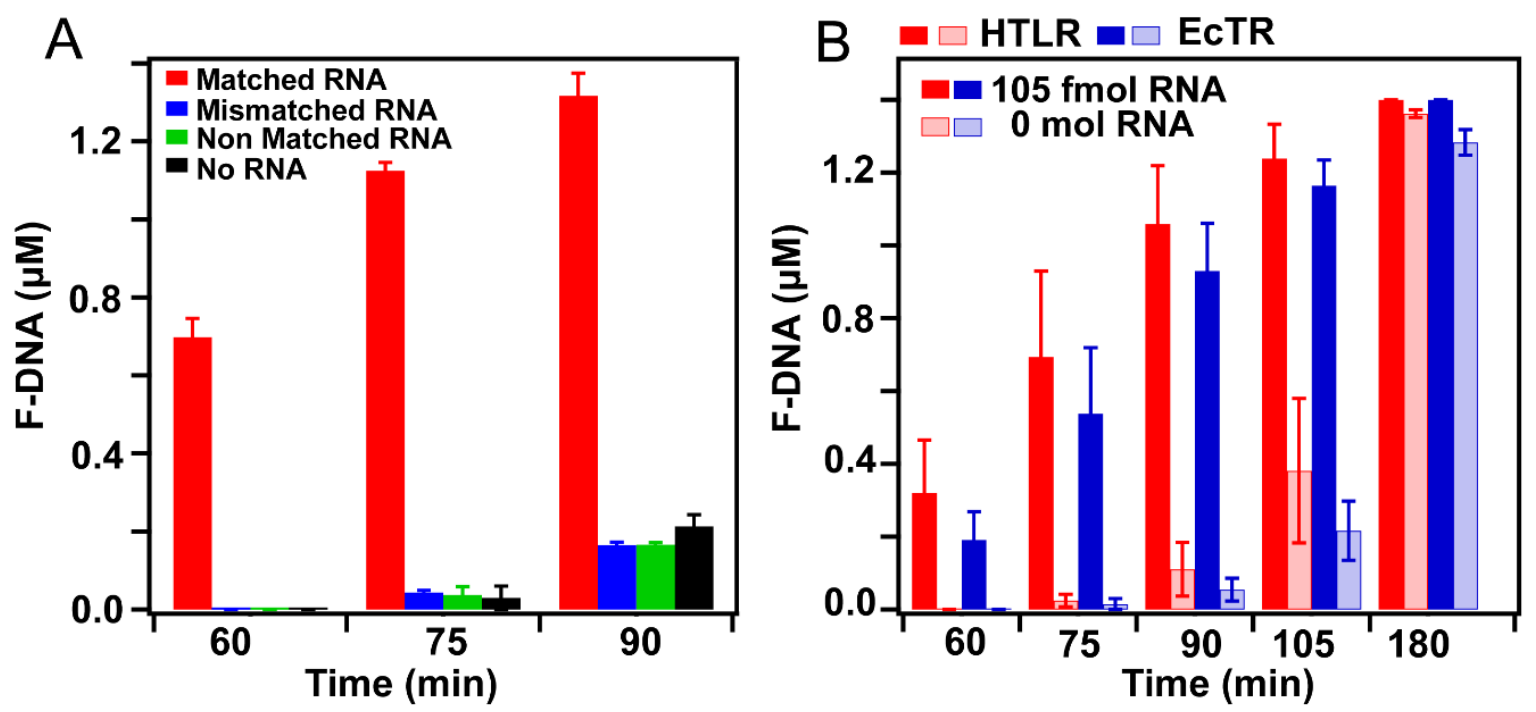

Figure 3. Specificity of reverse transcription LIDA. A) Formation of F-DNA initiated by various targets represented in red, blue and green after $60 \mathrm{~min}, 75 \mathrm{~min}$ and $90 \mathrm{~min}$ time point of the LIDA reaction. B) Formation of F-DNA initiated by $105 \mathrm{fmol}$ matched RNA (solid trace) and 0 mol matched RNA (faint trace) in the presence of $0.27 \mu \mathrm{g} / \mu \mathrm{L}$ human lungs total RNA (red traces) or E. coli total RNA (blue traces) at various time points.

\subsection{Detection of RNA target spiked in total RNA samples}

Biological fluids such as blood, saliva and urine are typically used for detection of diseases. [12, 13, 17] The cells in those samples are purified and lysed to collect cellular extract that contains the target RNA as well as all of the cellular RNA. Therefore, to determine the capability of our assay in detecting target RNA in the presence of realistic amounts of other RNA sequences, we conducted RT-LIDA in the presence of human lungs total RNA (HLTR) and E. coli total RNA (EcTR) to mimic the complexity of biological samples. Specifically, $105 \mathrm{fmol}$ of matched target RNA was spiked into both the HLTR and EcTR $(4 \mu \mathrm{g} / 15 \mu \mathrm{L})$. As seen in Figure $3 \mathrm{~B}$, at this target concentration, the reaction was not hindered by the presence of total RNA for either sample. Also, both the RNA target-initiated (solid red and blue bar) and the background-triggered (faint red and blue bars) reaction were the same within error despite the total RNA being from two completely different cell types. As mentioned previously, to increase the sensitivity 
of LIDA by further suppressing the background-triggered process allowing us to detect lower initial target concentrations, we typically lower the probe concentration. However, we observed that when we performed RT-LIDA at these lower probe concentrations with sample spiked in a pool of random RNA, LIDA was slowed down significantly (Figure S6A). Therefore, we decided to use a higher concentration of probes to identify the limit of detection in spiked samples (Figure S6B). Based on these conditions we could detect as low as $14 \mathrm{pM}$ target RNA in $4 \mu \mathrm{g} / 15 \mu \mathrm{L}$ of cellular RNA. This corresponds to the detection of $105 \mathrm{amol}$ in $4 \mu \mathrm{g}$ total RNA, which falls into the range of sensitivity required for real samples.[42-45] For example, Zhang reported that the amount of miR-21 in 8 cancerous tissue samples from patients having cervical cancer was $\sim 0.95-1.58 \mathrm{fmol} / \mu \mathrm{g} .[42]$

\subsection{RT-LIDA followed by colorimetric detection at room temperature}

Finally, to achieve instrument-free room-temperature colorimetric amplification and detection, we combined our colorimetric method previously reported by our group with RT-LIDA. This method is based on the rapid target-triggered disassembly of aggregated DNA-modified gold nanoparticles (AuNP),leading to a striking color change from blue precipitate to red solution.[36] Our previous work revealed that incorporating five single-stranded polyA regions at the $3^{\prime}$ - and 5'-termini of the linker DNA that held the AuNP together via hybridization allowed for rapid target-triggered disassembly by toehold mediated strand displacement over a range of temperatures that encompass room temperature including $28^{\circ} \mathrm{C}$. Consequently, probes were prepared that resulted in CDNA with 5 polyTs appended to the $3^{\prime}$ and $5^{\prime}$ ends, which would initiate toehold-mediated strand displacement (Figure 4A). Using these new probes, LIDA was performed with the optimized conditions on a sun-exposed lab bench. The temperature at that location recorded throughout the assay was $27-30^{\circ} \mathrm{C}$. After specific time points, $3 \mu \mathrm{L}$ of the amplification sample was aliquoted from tube (+) which was initiated by 105 fmol RNA target and tube (-) which was initiated by 0 mol RNA target into another set of microtubes containing aggregated DNA modified gold nanoparticles. These aggregates had been stored as lyophilized samples for approximately ten days on 
the bench top and were then rehydrated using $10 \mu \mathrm{L}$ of water immediately prior to use. As shown in Figure 4A, after 60 minutes of the LIDA step the aliquot from the sample containing RNA target led to disaggregation and rapid color change to red. On the other hand, the aliquot from the negative control led to no color change until the LIDA step had proceeded $75 \mathrm{~min}$, which was consistent with the amount of time required for the background reaction to trigger amplification of cDNA. Interestingly, we observed that the addition of just the probes that comprised the cDNA could trigger aggregate disassembly (data not shown). Fortunately, this probe-triggered process did not occur when both sets of probes were present presumably because of competition between the probes, which are complementary to one another, and the linker DNA sequence.
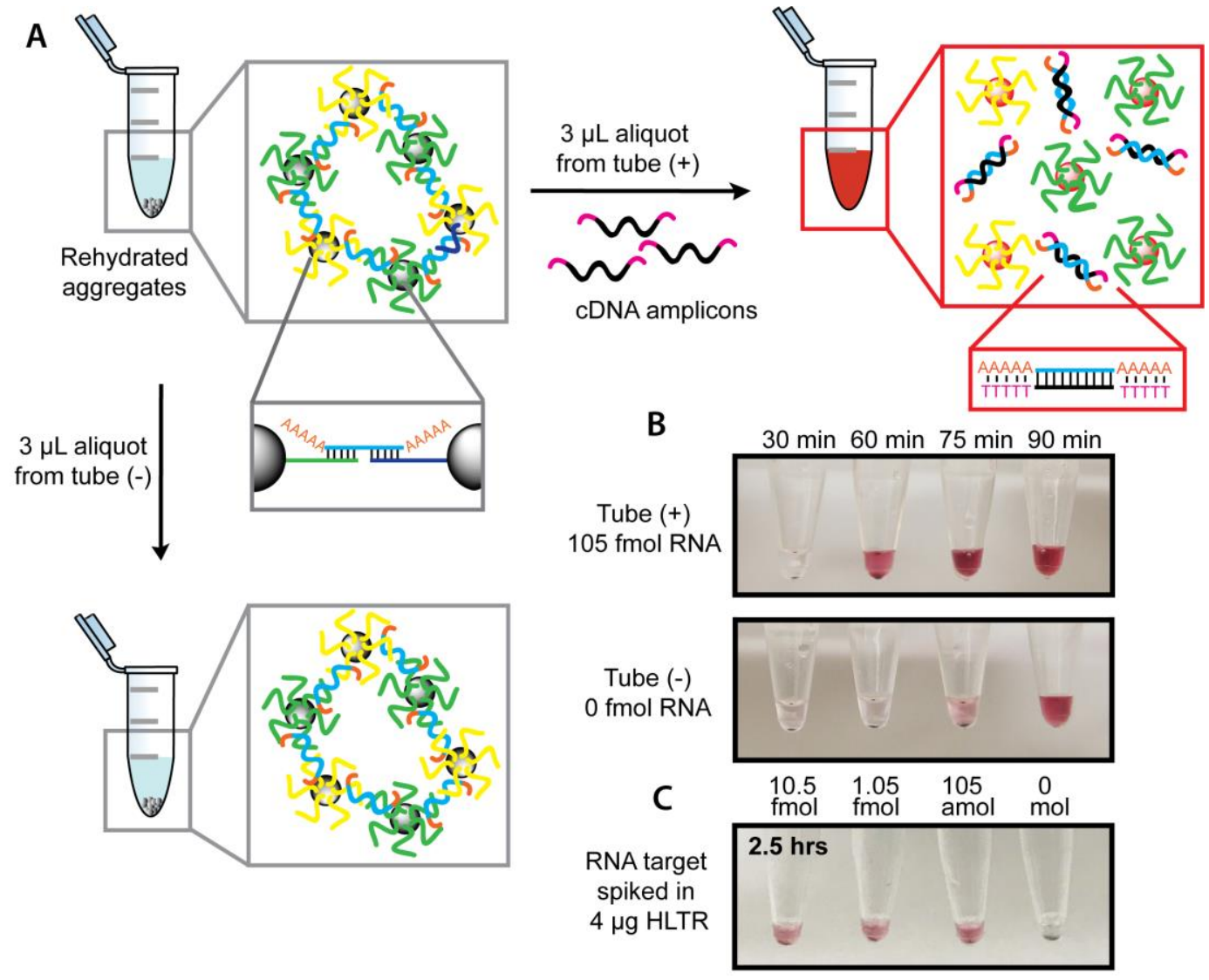

Figure 4. Room temperature colorimetric detection of cDNA amplicons using toe-hold mediated DNAmodified gold nanoparticle disassembly. A) Scheme representing toehold mediated target disassembly of 
AuNP triggered by the presence of cDNA. B) Image of DNA-AuNP aggregates after the addition of $3 \mu \mathrm{L}$ aliquots of the RT-LIDA reaction performed at room temperature initiated by $105 \mathrm{fmol}$ RNA (tube (+)) or 0 mol RNA (tube (-)) added at various time points. The picture was taken $15 \mathrm{~min}$ after adding the aliquot of the LIDA step to the aggregates. C) Images of reduced DNA-AuNP aggregates ( $1 / 3$ the amount shown in B) after 7.5 $\mu$ L aliquots of the RT-LIDA mixture initiated with various RNA target amounts were added after 2.5 hours amplification time. The picture was taken $15 \mathrm{~min}$ after adding the aliquot of the LIDA step to the aggregates. RT-LIDA in step C was performed in a thermal incubator at $28^{\circ} \mathrm{C}$.

Additionally, this colorimetric approach could successfully detect the cDNA amplificons in the presence of mismatched and random RNA sequences under conditions similar to those shown in Figure 3 (Figure S5). However, to achieve more sensitive detection, we reduced the amount of aggregates used in our visualization step. Under these conditions, 3 pmol of cDNA led to a clear color change while $2 \mathrm{pmol}$ did not (Figure S11A). Using these smaller aggregate samples, we could successfully detect 105 amol (14 pM) of target RNA after 2.5 hours of amplification in the presence of cellular RNA (Figure 4C), thereby achieving the same sensitivity as our point-of-inflection analysis using PAGE to monitor the reaction profiles. Having a colorimetric read-out, however, is far superior for POC applications and only requires 15 minutes to perform.

\subsection{Conclusion}

In summary, this work demonstrated that RNA can be amplified and detected isothermally without instrumentation by combining a reverse transcription step with our lesion induced DNA amplification system. Adding an RNA strand displacement step and a mismatch to LIDA allowed us to lower the assay temperature to $28^{\circ} \mathrm{C}$ and perform it on the bench in our lab. Furthermore, incorporating target-triggered disaggregation of gold nanoparticles resulted in colorimetric visualization of the target without requiring any heating element. A detection range of $14 \mathrm{pM}$ to $14 \mathrm{nM}$ with a limit of detection of $32 \mathrm{pM}$ ( 240 amol) was achieved based on an analysis of the point-of-inflection of kinetic amplification traces. However, we 
were visually able to discriminate $14 \mathrm{pM}$ (105 amol) of target RNA based on the colorimetric read-out. Our assay is simple, instrument-free and utilizes a small number of reagents and simple reaction vessels (eppendorf tubes). Moreover, this process is ideal for the detection of short strands of RNA like miRNA (18- 23 bases) providing an advantage to other techniques like RT-PCR where amplifying short sequences compromises the sensitivity and the specificity of the assay. Future work will address incorporating instrument-free methods for sample preparation including RNA isolation from biological samples.

\section{Declaration of competing interest}

The authors declare that they have no known competing financial interests or personal relationships that could have appeared to influence the work reported in this paper.

\section{Acknowledgements}

JMG gratefully acknowledges the Alfred P. Sloan Foundation for a Research Fellowship, Grand Challenges

Canada for a Rising Star Award, Future Energy Systems and an Natural Sciences and Engineering Research Council of Canada (NSERC) for an Accelerator Award. BSA-M gratefully acknowledges the Alberta Innovates Technology Futures for a graduate scholarship. Y Liu gratefully acknowledges the Alberta/Technical University of Munich Graduate School for Hybrid Functional Materials (ATUMS-NSERC CREATE). We thank Gareth Lambkin for guidance and the U Alberta, Dept of Chemistry, Biological Services Facility for use of equipment.

\section{Supplementary data}

Electronic supplementary information available.

\section{References}

[1] X. Xi, T. Li, Y. Huang, J. Sun, Y. Zhu, Y. Yang, Z. Lu, RNA Biomarkers: Frontier of Precision Medicine for Cancer, Non-Coding RNA 3(1) (2017) 9.

[2] J.F. Quinn, T. Patel, D. Wong, S. Das, J.E. Freedman, L.C. Laurent, B.S. Carter, F. Hochberg, K.V. Keuren-Jensen, M. Huentelman, R. Spetzler, M.Y.S. Kalani, J. Arango, P.D. Adelson, H.L. Weiner, R. Gandhi, B. Goilav, C. Putterman, J.A. Saugstad, Extracellular RNAs: development as biomarkers of human disease, Journal of Extracellular Vesicles 4 (2015) 10.3402/jev.v4.27495. 
[3] L.N. Cella, D. Blackstock, M.A. Yates, A. Mulchandani, W. Chen, Detection of RNA viruses: current technologies and future perspectives, Crit Rev Eukaryot Gene Expr 23(2) (2013) 125-37.

[4] B. Koo, C.E. Jin, T.Y. Lee, J.H. Lee, M.K. Park, H. Sung, S.Y. Park, H.J. Lee, S.M. Kim, J.Y. Kim, S.H. Kim, Y. Shin, An isothermal, label-free, and rapid one-step RNA amplification/detection assay for diagnosis of respiratory viral infections, Biosens Bioelectron 90 (2017) 187-194.

[5] A. Priye, S.W. Bird, Y.K. Light, C.S. Ball, O.A. Negrete, R.J. Meagher, A smartphone-based diagnostic platform for rapid detection of Zika, chikungunya, and dengue viruses, Scientific Reports 7 (2017) 44778. [6] L. Dietz, P. Horve, D. Coil, M. Fretz, K. Wymelenberg, 2019 Novel Coronavirus (COVID-19) Outbreak: A Review of the Current Literature and Built Environment (BE) Considerations to Reduce Transmission, 2020.

[7] W. Xia, J. Shao, Y. Guo, X. Peng, Z. Li, D. Hu, Clinical and CT features in pediatric patients with COVID19 infection: Different points from adults, Pediatric Pulmonology 55(5) (2020) 1169-1174.

[8] Z. Dvorak, J.M. Pascussi, M. Modriansky, Approaches to messenger RNA detection - comparison of methods, Biomed Pap Med Fac Univ Palacky Olomouc Czech Repub 147(2) (2003) 131-5.

[9] N. Rijpens, G. Jannes, L. Herman, Messenger RNA-based RT-PCR detection of viable Salmonella, International Dairy Journal 12(2) (2002) 233-238.

[10] M.N. Islam, V. Gopalan, M.H. Haque, M.K. Masud, M.S.A. Hossain, Y. Yamauchi, N.T. Nguyen, A.K. Lam, M.J.A. Shiddiky, A PCR-free electrochemical method for messenger RNA detection in cancer tissue samples, Biosens Bioelectron 98 (2017) 227-233.

[11] J. Fan, X. Zhang, Y. Cheng, C. Xiao, W. Wang, X. Liu, C. Tong, B. Liu, Increasing the sensitivity and selectivity of a GONS quenched probe for an mRNA assay assisted with duplex specific nuclease, RSC Advances 7(57) (2017) 35629-35637.

[12] H. Dong, J. Lei, L. Ding, Y. Wen, H. Ju, X. Zhang, MicroRNA: Function, Detection, and Bioanalysis, Chemical Reviews 113(8) (2013) 6207-6233.

[13] L. Yan, J. Zhou, Y. Zheng, A.S. Gamson, B.T. Roembke, S. Nakayama, H.O. Sintim, Isothermal amplified detection of DNA and RNA, Mol Biosyst 10(5) (2014) 970-1003.

[14] W. Wang, T. Kong, D. Zhang, J. Zhang, G. Cheng, Label-Free MicroRNA Detection Based on Fluorescence Quenching of Gold Nanoparticles with a Competitive Hybridization, Anal Chem 87(21) (2015) 10822-9.

[15] Y. Tian, L. Zhang, H. Wang, W. Ji, Z. Zhang, Y. Zhang, Z. Yang, Z. Cao, S. Zhang, J. Chang, Intelligent Detection Platform for Simultaneous Detection of Multiple MiRNAs Based on Smartphone, ACS Sensors 4(7) (2019) 1873-1880.

[16] M. Marmot, Social determinants of health inequalities, Lancet 365(9464) (2005) 1099-104.

[17] P. Craw, W. Balachandran, Isothermal nucleic acid amplification technologies for point-of-care diagnostics: a critical review, Lab Chip 12(14) (2012) 2469-86.

[18] J. Song, M.G. Mauk, B.A. Hackett, S. Cherry, H.H. Bau, C. Liu, Instrument-Free Point-of-Care Molecular Detection of Zika Virus, Anal Chem 88(14) (2016) 7289-94.

[19] L.K. Lafleur, J.D. Bishop, E.K. Heiniger, R.P. Gallagher, M.D. Wheeler, P. Kauffman, X. Zhang, E.C. Kline, J.R. Buser, S. Kumar, S.A. Byrnes, N.M.J. Vermeulen, N.K. Scarr, Y. Belousov, W. Mahoney, B.J. Toley, P.D. Ladd, B.R. Lutz, P. Yager, A rapid, instrument-free, sample-to-result nucleic acid amplification test, Lab on a Chip 16(19) (2016) 3777-3787.

[20] P. LaBarre, D. Boyle, K. Hawkins, B. Weigl, Instrument-free nucleic acid amplification assays for global health settings, Proc SPIE Int Soc Opt Eng 8029 (2011).

[21] D. Mabey, R.W. Peeling, A. Ustianowski, M.D. Perkins, Diagnostics for the developing world, Nat Rev Microbiol 2(3) (2004) 231-40. 
[22] K. Zhang, K. Wang, X. Zhu, M. Xie, A one-pot strategy for the sensitive detection of miRNA by catalyst-oligomer-mediated enzymatic amplification-based fluorescence biosensor, Sensors and Actuators B: Chemical 223 (2016) 586-590.

[23] S. Hwu, Y. Blickenstorfer, R.F. Tiefenauer, C. Gonnelli, L. Schmidheini, I. Luchtefeld, B.J. Hoogenberg, A.B. Gisiger, J. Voros, Dark-Field Microwells toward High-Throughput Direct miRNA Sensing with Gold Nanoparticles, ACS Sens 4(7) (2019) 1950-1956.

[24] Y. Shen, F. Tian, Z. Chen, R. Li, Q. Ge, Z. Lu, Amplification-based method for microRNA detection, Biosensors and Bioelectronics 71 (2015) 322-331.

[25] Y. Sun, H. Tian, C. Liu, Y. Sun, Z. Li, One-step detection of microRNA with high sensitivity and specificity via target-triggered loop-mediated isothermal amplification (TT-LAMP), Chem Commun (Camb) 53(80) (2017) 11040-11043.

[26] X. Li, M. Ni, C. Zhang, W. Ma, Y. Zhang, A convenient system for highly specific and sensitive detection of miRNA expression, Rna 20(2) (2014) 252-9.

[27] P. Chugh, D.P. Dittmer, Potential pitfalls in microRNA profiling, Wiley Interdiscip Rev RNA 3(5) (2012) 601-16.

[28] P. Lam, R.A. Keri, N.F. Steinmetz, A Bioengineered Positive Control for Rapid Detection of the Ebola Virus by Reverse Transcription Loop-Mediated Isothermal Amplification (RT-LAMP), ACS Biomaterials Science \& Engineering 3(3) (2017) 452-459.

[29] H. Xu, D. Wu, Y. Zhang, H. Shi, C. Ouyang, F. Li, L. Jia, S. Yu, Z.-S. Wu, RCA-enhanced multifunctional molecule beacon-based strand-displacement amplification for sensitive microRNA detection, Sensors and Actuators B: Chemical 258 (2018) 470-477.

[30] J. Zhuang, W. Lai, G. Chen, D. Tang, A rolling circle amplification-based DNA machine for miRNA screening coupling catalytic hairpin assembly with DNAzyme formation, Chem Commun (Camb) 50(22) (2014) 2935-8.

[31] D.-M. Zhou, W.-F. Du, Q. Xi, J. Ge, J.-H. Jiang, Isothermal Nucleic Acid Amplification Strategy by Cyclic Enzymatic Repairing for Highly Sensitive MicroRNA Detection, Analytical Chemistry 86(14) (2014) 6763-6767.

[32] S. Fang, H.J. Lee, A.W. Wark, R.M. Corn, Attomole Microarray Detection of MicroRNAs by Nanoparticle-Amplified SPR Imaging Measurements of Surface Polyadenylation Reactions, Journal of the American Chemical Society 128(43) (2006) 14044-14046.

[33] B.S. Alladin-Mustan, C.J. Mitran, J.M. Gibbs-Davis, Achieving room temperature DNA amplification by dialling in destabilization, Chemical Communications 51(44) (2015) 9101-9104.

[34] A. Kausar, R.D. McKay, J. Lam, R.S. Bhogal, A.Y. Tang, J.M. Gibbs-Davis, Tuning DNA Stability To Achieve Turnover in Template for an Enzymatic Ligation Reaction, Angewandte Chemie International Edition 50(38) (2011) 8922-8926.

[35] A. Kausar, C.J. Mitran, Y. Li, J.M. Gibbs-Davis, Rapid, isothermal DNA self-replication induced by a destabilizing lesion, Angew Chem Int Ed Engl 52(40) (2013) 10577-81.

[36] M.K. Lam, T. Gadzikwa, T. Nguyen, A. Kausar, B.S. Alladin-Mustan, M.D. Sikder, J.M. Gibbs-Davis, Tuning Toehold Length and Temperature to Achieve Rapid, Colorimetric Detection of DNA from the Disassembly of DNA-Gold Nanoparticle Aggregates, Langmuir 32(6) (2016) 1585-1590.

[37] G. Ko, T.L. Cromeans, M.D. Sobsey, Detection of infectious adenovirus in cell culture by mRNA reverse transcription-PCR, Appl Environ Microbiol 69(12) (2003) 7377-84.

[38] K. Zhang, K. Wang, X. Zhu, F. Xu, M. Xie, Sensitive detection of microRNA in complex biological samples by using two stages DSN-assisted target recycling signal amplification method, Biosensors and Bioelectronics 87 (2017) 358-364. 
[39] B.-C. Yin, Y.-Q. Liu, B.-C. Ye, One-Step, Multiplexed Fluorescence Detection of microRNAs Based on Duplex-Specific Nuclease Signal Amplification, Journal of the American Chemical Society 134(11) (2012) 5064-5067.

[40] W. Du, M. Lv, J. Li, R. Yu, J. Jiang, A ligation-based loop-mediated isothermal amplification (ligationLAMP) strategy for highly selective microRNA detection, Chemical Communications 52(86) (2016)

12721-12724.

[41] M. Nilsson, D.-O. Antson, G. Barbany, U. Landegren, RNA-templated DNA ligation for transcript analysis, Nucleic Acids Research 29(2) (2001) 578-581.

[42] W. Zhang, P. Zhang, F. Zhang, W. Cheng, Y. Xu, Y. Zhang, L. Chen, H. Wang, Q. Zhou, X. Zhang, Realtime and rapid quantification of microRNAs in cells and tissues using target-recycled enzyme-free amplification strategy, Talanta 217 (2020) 121016.

[43] S. Li, L. Xu, W. Ma, X. Wu, M. Sun, H. Kuang, L. Wang, N.A. Kotov, C. Xu, Dual-Mode Ultrasensitive Quantification of MicroRNA in Living Cells by Chiroplasmonic Nanopyramids Self-Assembled from Gold and Upconversion Nanoparticles, Journal of the American Chemical Society 138(1) (2016) 306-312. [44] F. Degliangeli, P. Kshirsagar, V. Brunetti, P.P. Pompa, R. Fiammengo, Absolute and Direct MicroRNA Quantification Using DNA-Gold Nanoparticle Probes, Journal of the American Chemical Society 136(6) (2014) 2264-2267.

[45] X. Zhao, L. Xu, M. Sun, W. Ma, X. Wu, H. Kuang, L. Wang, C. Xu, Gold-Quantum Dot Core-Satellite Assemblies for Lighting Up MicroRNA In Vitro and In Vivo, Small $12(34)$ (2016) 4662-4668. 